\title{
Design and analysis of compact dual resonance patch antenna
}

\author{
Amina Puran ${ }^{1 *}$, Şehabeddin Taha İmeci ${ }^{2}$ \\ ${ }^{1,2}$ Department of Electrical and Electronics Engineering, International University of Sarajevo, Bosnia
}

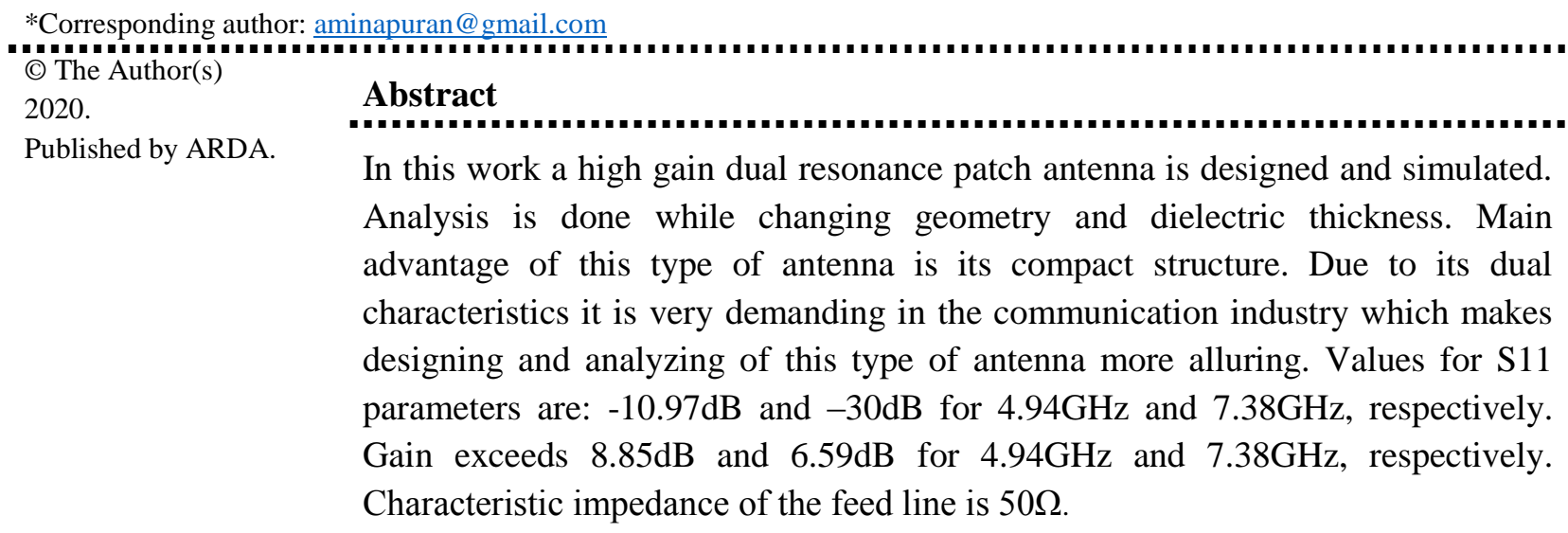

Keywords: Microstrip patch antenna, Microstrip feed line, Radiation pattern, Via fed

\section{Introduction}

Antenna is an important part of the communication system. The performance of the communication link depends upon the performance of the antenna. Microstrip patch antenna is compact, low-cost and usually excels has high efficiency.

Its geometry consists of the ground plane and radiating patch with dielectric substrate between them. Designed antenna shown in figure 1 has high gain and low input impedance which makes it efficient and profitable.

These advantages make them outstanding when it comes to the communication industry, as part of satellites, remote sensing systems and radars [1].

The modern research on this type of the antennas is used to assist the curing of breast cancer. Different approaches such as arrays of the microstrip patch antennas are used to improve efficiency of the device. Mostly arrays are used to perform the functions which one single element can't do and to increase the directivity and return loss [2].

The size of the antenna which is analyzed and designed in this paper together with other characteristics is given as follows:

a) box size: $500 \mathrm{~mm} \times 500 \mathrm{~mm}$;

b) cell size: $0.8 \mathrm{~mm}$;

c) frequency range: $4 \mathrm{GHz}$ to $9 \mathrm{GHz}$.

Design and analysis are obtained by using sonnet software [3]. 


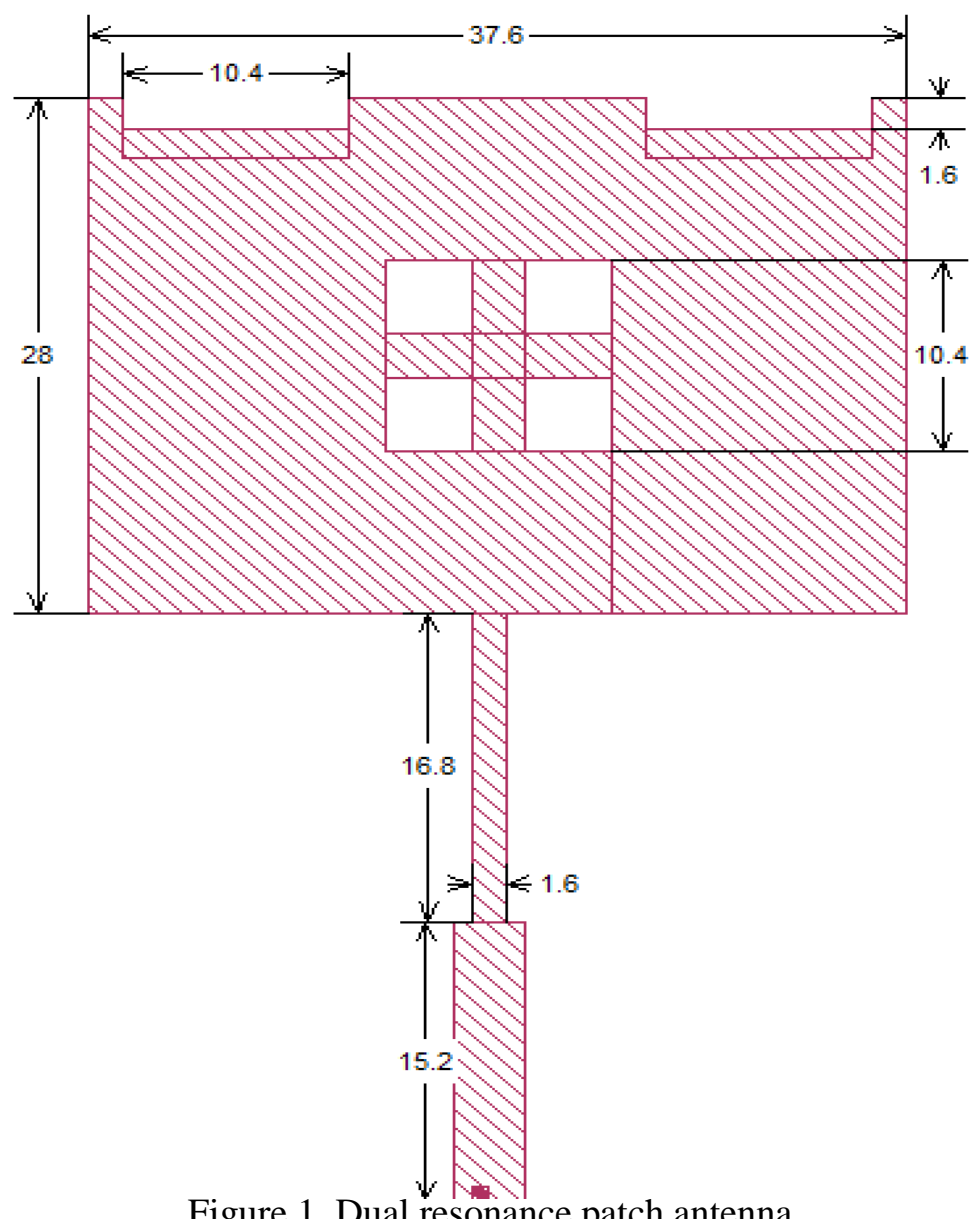

Figure 1. Dual resonance patch antenna

\section{Design methodology}

Dual characteristic is obtained by compilation of rectangular slits as can be seen from figure 1 . While the main role in improving the performance of the antenna had a gap with cross at the center which also can be seen in figure 1. Dimensions of the antenna represented in figure 1 show the compactness of it and note that all of the dimensions are given in $\mathrm{mm}$. Via feeding method is used in order to increase overall performance of the antenna and to simplify manufacturing of the antenna. Output signals are shown in figure 2 (S11) and figure 3 (gain). While designing we aim for the high absolute value of the input match (s11) since it represents the measure of the power absorbed by load.

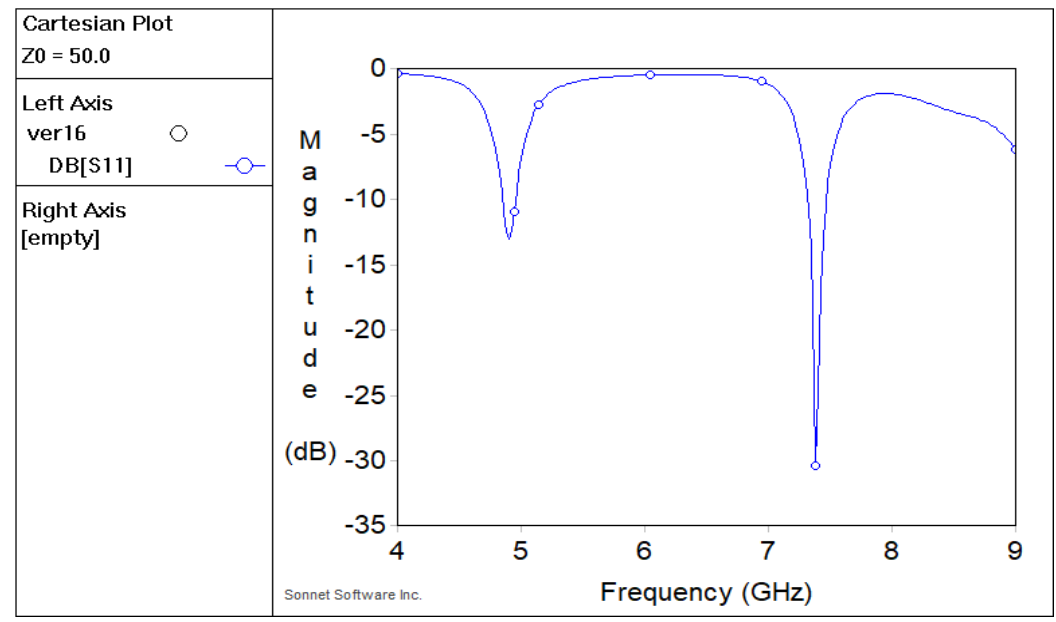

Figure 2. Input match response of dual resonance patch antenna 
Gain is the representation of the power density concentrated in a particular direction [4]. It is alluded to the isotropic reference antenna which radiates equally to all directions. Further, gain is given in the dB.

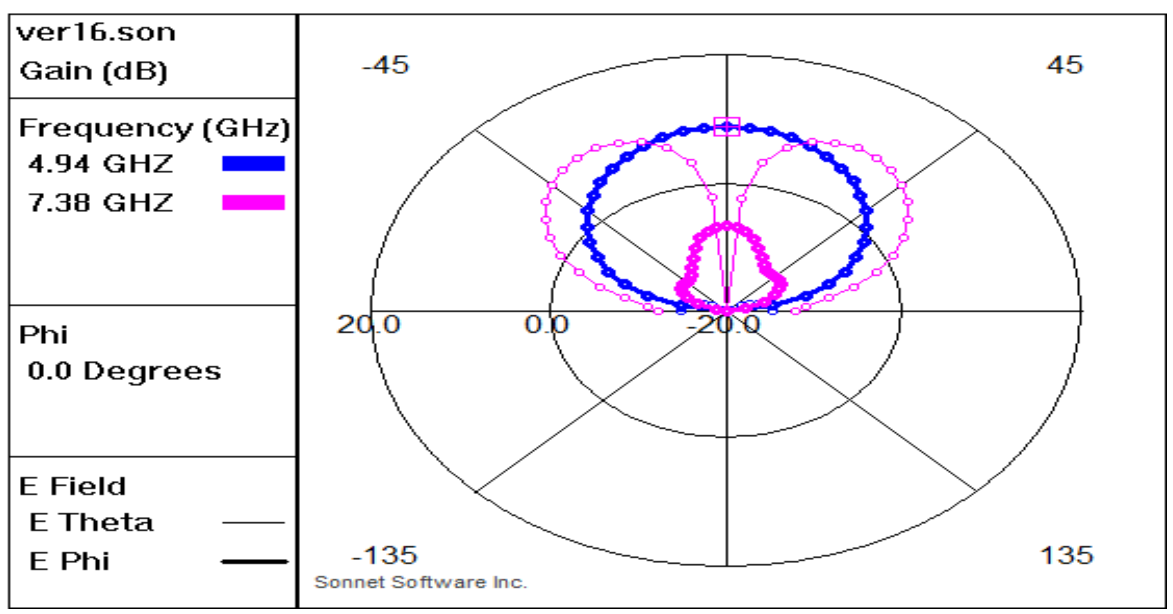

Figure 3. Gain response of dual resonance patch antenna

\section{Parameters variations}

In order to find optimum design adjustments that includes geometry dielectric thickness are done. Analysis on the dual resonance patch antenna includes changing the width of the gap with cross inside (Table 1), slit's length changes (Table 2), dielectric thickness (Table 3 and 4) and port location (Table 5).

We observed the frequency at which S11 magnitude and gain occurs. From Table 1 we can conclude that the best performance is for a gap width of $10.4 \mathrm{~mm}$.

Table 1. Gap width changes

\begin{tabular}{llll}
\hline WIDTH $(\mathrm{mm})$ & Frequency $(\mathrm{GHz})$ & S11 $(\mathrm{dB})$ & Gain $(\mathrm{dB})$ \\
\hline 7.2 & 5.04 & -16.47 & 8.88 \\
& 7.42 & -19.32 & 5.71 \\
8.8 & 4.98 & -15.48 & 8.82 \\
& 7.42 & -22.01 & 5.92 \\
10.4 & 4.94 & -10.97 & 8.85 \\
& 7.38 & -30.33 & 6.59 \\
12 & 4.8 & -10.73 & 8.56 \\
& 7.32 & -24.07 & 7.08 \\
13.6 & 4.74 & -8.71 & 8.39 \\
& 7.12 & -17.26 & 6.13 \\
\hline
\end{tabular}

Table 2 shows the changes of the slits length. They are located at the right and left upper corner of the antenna. Slits on both sides are perfectly symmetrical and work as a pair which means that changes in one slit reproduce change on another slit. Slit width of $1.6 \mathrm{~mm}$ gives the best performance. 
Table 2. Slit length changes

\begin{tabular}{llll}
\hline LENGTH $(\mathrm{mm})$ & Frequency $(\mathrm{GHz})$ & S11 $(\mathrm{dB})$ & Gain $(\mathrm{dB})$ \\
\hline 0.8 & 4.88 & -10.99 & 8.76 \\
& 7.48 & -23.25 & 7.28 \\
1.6 & 4.94 & -10.97 & 8.85 \\
& 7.38 & -30.33 & 6.59 \\
2.4 & 4.96 & -13.35 & 8.81 \\
& 7.24 & -23.50 & 5.25 \\
3.2 & 4.98 & -13.77 & 8.77 \\
& 7.06 & -18.41 & 3.15 \\
4 & 5.02 & -14.86 & 8.81 \\
& 6.82 & -13.94 & 2.19 \\
\hline
\end{tabular}

Changing of the dielectric thickness is shown in Table 3. Dielectric thickness of 20 produces best performance but it is too thick for microstrip antennas. With all trade off we concluded that the best dielectric thickness for this design is $10 \mathrm{~mm}$.

Table 3. Changing dielectric thickness 1

\begin{tabular}{llll}
\hline $\begin{array}{l}\text { Dielectric } \\
(\mathrm{mm})\end{array}$ & thickness Frequency $(\mathrm{GHz})$ & S11 $(\mathrm{dB})$ & Gain $(\mathrm{dB})$ \\
\hline 5 & 4.92 & -20.45 & 6.45 \\
& 7.28 & -15.26 & 5.30 \\
10 & 4.94 & -10.97 & 8.85 \\
& 7.38 & -30.33 & 6.59 \\
15 & 4.92 & -10.00 & 9.55 \\
& 7.38 & -24.32 & 7.04 \\
20 & 4.92 & -9.54 & 10.44 \\
& 7.38 & -19.88 & 7.48 \\
\hline
\end{tabular}

However, in order to have a practical microstrip antenna we changed the dielectric thickness of the antenna in the range of $1 \mathrm{~mm}$ to $3 \mathrm{~mm}$. Results are given in Table 4 . We also achieved triple response in these simulations.

Table 4. Changing dielectric thickness 2

\begin{tabular}{llll}
\hline Dielectric thickness $(\mathrm{mm})$ & Frequency $(\mathrm{GHz})$ & $\mathrm{S} 11(\mathrm{~dB})$ & Gain $(\mathrm{dB})$ \\
\hline 0.5 & 4.92 & -42.49 & 6.25 \\
& 7.88 & -3.55 & 0.15 \\
& 9.0 & -8.14 & 4.59 \\
1 & 4.94 & -10.97 & 8.85 \\
& 7.38 & -30.34 & 6.59 \\
& 9.99 & -6.20 & 6.93 \\
1.5 & 4.84 & -10.89 & 9.37 \\
& 8.38 & -4.60 & 29.04
\end{tabular}




\begin{tabular}{llll}
2.5 & 9.00 & -7.46 & 1.91 \\
& 4.94 & -10.11 & 10.13 \\
& 8.32 & -10.67 & 0.51 \\
3 & 9.0 & -14.95 & 18.20 \\
& 4.98 & -9.26 & 10.18 \\
& 8.34 & -12.13 & 2.80 \\
& 9.0 & -14.25 & 13.93 \\
\hline
\end{tabular}

Port location is very important to achieve good directivity of the antenna. Further, it shows the best position of the port for fabrications.

Up, left and right port locations represent middle positions in the antenna upper rectangle while bottom is shown in figure 1.

From Table 5 we can conclude that port location at bottom gives best performance regarding both S11 and gain.

Table 5. Changing port location

\begin{tabular}{llll}
\hline Port Location & Frequency $(\mathrm{GHz})$ & S11 $(\mathrm{dB})$ & Gain $(\mathrm{dB})$ \\
\hline UP & 6.62 & -5.11 & 6.52 \\
& 7.88 & -7.51 & 11.47 \\
LEFT & 6.66 & -16.68 & 7.30 \\
\multirow{2}{*}{ RIGHT } & 7.42 & -15.23 & 9.99 \\
& 6.66 & -16.45 & 7.29 \\
& 7.42 & -16.91 & 9.94 \\
BOTTOM & 8.06 & -12.53 & 0.15 \\
& 4.94 & -10.97 & 8.85 \\
& 7.38 & -30.33 & 6.59 \\
\hline
\end{tabular}

Before we reached results shown in Tables 1, 2, 3 and 4 design changes have been done. They are mostly based on changing the shape of the slit and obtained results are given in Table 6. However, we did not expect to have dual resonance so we did analysis in frequency range 1 to 5 .

Table 6. Design changes on frequency range 1 to 5

\begin{tabular}{|c|c|c|c|}
\hline Changing the slit shape & Frequency $(\mathrm{GHz})$ & $\mathrm{S} 11(\mathrm{~dB})$ & Gain $(\mathrm{dB})$ \\
\hline RECTANGULAR & 5.04 & -14.75 & 6.40 \\
\hline $\begin{array}{l}\text { RECTANGULAR W } \\
\text { TWO GAPS }\end{array}$ & VITH 5.10 & -17.31 & 6.40 \\
\hline STAR & 4.82 & -9.35 & 5.36 \\
\hline STAR & VITH 4.84 & -10.05 & 5.39 \\
\hline \multicolumn{4}{|l|}{ ADDITIONAL GAP } \\
\hline SQUARE & 4.86 & -10.27 & 5.46 \\
\hline
\end{tabular}


After finding which shape gives best results, we have analyzed how the cell size affects the antenna performance. Results of this analysis are given in Table 7.

Table 7. Changing the cell size

\begin{tabular}{llll}
\hline Cell size & Frequency $(\mathrm{GHz})$ & $\mathrm{S} 11(\mathrm{~dB})$ & Gain $(\mathrm{dB})$ \\
\hline 0.6 & 5.24 & -13.24 & 6.98 \\
0.7 & 5.08 & -21.25 & 6.65 \\
0.8 & 4.96 & -12.25 & 5.96 \\
0.9 & 4.86 & -11.61 & 5.9 \\
& 7.38 & -30.33 & 6.59 \\
\hline
\end{tabular}

In order to achieve the best antenna performance and do other analysis on specific types we chose to change the width of the port/via carrier. It is included as one part of design changes. Theory says that the wider it gets the better performance we achieve. In Table 8 are shown results we obtained.

Table 8. Port/via carrier width

\begin{tabular}{llll}
\hline Width carrier & Frequency $(\mathrm{GHz})$ & $\mathrm{S} 11(\mathrm{~dB})$ & Gain $(\mathrm{dB})$ \\
\hline 1.6 & 4.68 & -13.54 & 6.12 \\
1.7 & 4.94 & -12.62 & 6.04 \\
1.8 & 5.08 & -15.11 & 6.47 \\
1.9 & 4.96 & -9.08 & 8.88 \\
2.0 & 4.86 & -16.96 & 8.36 \\
\hline
\end{tabular}

We also performed analysis on changing the dielectric thickness from $1 \mathrm{~mm}$ to $3 \mathrm{~mm}$ on different designs. The one with best performance in that range is selected to be represented in Table 3 and 4. Designs are the same as one given in Table 5. We also increased the frequency range to be from $4 \mathrm{GHz}$ to $9 \mathrm{GHz}$. Results obtained are shown in Table 9.

Table 9. Changing dielectric thickness on different shapes

\begin{tabular}{|c|c|c|c|}
\hline Changing the slit shape & Frequency $(\mathrm{GHz})$ & $\mathrm{S} 11(\mathrm{~dB})$ & Gain $(\mathrm{dB})$ \\
\hline \multirow[t]{2}{*}{ RECTANGULAR } & 4.84 & -10.89 & 9.37 \\
\hline & 9.0 & -7.46 & 29.04 \\
\hline \multirow{2}{*}{$\begin{array}{l}\text { RECTANGULAR W } \\
\text { TWO GAPS }\end{array}$} & 4.83 & -4.12 & 9.53 \\
\hline & 8.51 & -6.54 & 6.34 \\
\hline \multirow[t]{2}{*}{ STAR } & 4.92 & -12.11 & 4.87 \\
\hline & 7.89 & -9.72 & 11.24 \\
\hline STAR & 5.14 & -3.12 & 16.38 \\
\hline \multicolumn{4}{|c|}{ ADDITIONAL GAP } \\
\hline & 9.1 & -19.76 & 2.34 \\
\hline \multirow[t]{2}{*}{ SQUARE } & 4.58 & -8.19 & 8.13 \\
\hline & 8.68 & -19.94 & 3.82 \\
\hline
\end{tabular}




\section{Numerical analysis and equivalent circuit}

In order to predict and produce valuable results numerical analysis should be done. Behind the design of the antenna there are equations which explain how the antenna operates. Radiation is represented by the E field [5]. Radiating plane is composed of electrical and magnetic waves. There are various types of polarization as follows: linear, circular and elliptical. From names we can conclude that it depends upon the shape of radiation waves radiating from the antenna. Equations for the E-field for linear, circular and elliptical are given in equation 1,2 and 3 respectively.

$$
\begin{gathered}
E=\cos \left(2 \pi \boldsymbol{f}\left(\boldsymbol{t}-\frac{z}{c}\right)\right)(\hat{x}+\hat{y}) \\
E=\cos \left(2 \pi \boldsymbol{f}\left(\boldsymbol{t}-\frac{z}{c}\right)\right) \hat{x}+\sin \left(2 \pi \boldsymbol{f}\left(\boldsymbol{t}-\frac{z}{c}\right)\right) \hat{y} \\
E=\cos \left(2 \pi \boldsymbol{f}\left(\boldsymbol{t}-\frac{z}{c}\right)\right) \hat{x}+0.3 \sin \left(2 \pi \boldsymbol{f}\left(\boldsymbol{t}-\frac{z}{c}\right)\right) \hat{y}
\end{gathered}
$$

In equations vector values represent direction of the $\mathrm{E}$ field. In linear polarization propagation of the $\mathrm{E}$ field stays at one single line. Field can be either horizontally or vertically propagated depending upon the $\mathrm{x}$ axis position to the ground. However, there are cases where radiation is in one positioned at one single line but the E field is not propagating either $\mathrm{x}$ or $\mathrm{y}$ axis. Those cases still represent linear radiation. As can be seen in equation $1 \mathrm{x}$ and $\mathrm{y}$ components are in phase and have the same magnitude. Equation 2 represents the value of the magnetic field for circular radiation. Components $\mathrm{x}$ and $\mathrm{y}$ are 90 degrees out of phase and the E field rotates in a cycle so it gets name circular. Further, components must have the same magnitude and be orthogonal to each other. The direction depends on the direction of rotation of the E-field. It can be clockwise or counter clockwise. For elliptical polarization components don't have equal magnitude and are out of phase for 90 degrees. Waves in this type of polarization are defined by a major axis. Axial ratio, the ratio between major and minor axis amplitudes, is one of the ways to represent the polarization [6]. If the axial ratio is 1 it is a circularly polarized wave while infinite axial ratio represents a linearly polarized wave. Any value between it two gives an elliptical wave. Due to the reciprocity theorem antennas transmit and receive in the same manner [7]. If the vertically polarized antenna transmits the signal, the vertically polarized antenna will receive signal. Any communication between opposite polarized antennas will not be successful. When two antennas are positioned from each other with angle $\theta$, the power loss factor is given in equation 4 .

$$
P L F=\cos ^{2} \theta
$$

For equation 4, if the angle $\theta$ is 0 it means that there is no power loss. However, if the antennas are opposite positioned (horizontally and vertically) the angle $\theta$ is 90 and there is no power transmitted. Power density needed to transmit the signal properly is defined in equation 5.

$$
P_{d} \quad=\frac{1}{2} R_{e}(\underline{E} \times \underline{H \quad \star})=\frac{E^{2}}{2 \eta_{o}}
$$

where, $\eta_{o}$ is the intrinsic impedance of the free space [8]. It represents the density in the wave needed to transmit signal from the antenna. In order to transmit the signal, there must be a reference antenna which is usually an isotropic antenna. It is the type of antenna which transmits equally to all directions [9]. Power density at any distance from the reference antenna is power radiated from the transmitter divided by the spherical surface area which is given in equation 6 [10].

$$
P_{d}=\frac{P_{t}}{4 \pi R^{2}}
$$

Further, one of the main parameters in tracking the performance of the antenna is antenna gain. It represents the directivity of the radiation of the antenna. Gain is given in equation 7. 


$$
G=\frac{P_{(\text {radiated by the antenna })}}{P_{(\text {radiated by refrence antenna })}}
$$

Far field is a region of radiation of electromagnetic fields around the object which consists of the magnetic and electric field. Electric and magnetic field strength decreases as distance of the object increases [11].

\section{Conclusion}

Design demonstrated in this paper represents a simple, affordable and compact device. It is an important part of the communication wireless technology. However, excitation of surface waves which occur in the substrate layer is a disadvantage of patch antenna [12]. Further, it is the main reason why choosing proper design and dielectric thickness is important. Simulations are done in one minute which manifests high speed response. Values for S11 parameters are: $-10.97 \mathrm{~dB}$ and $-30 \mathrm{~dB}$ for $4.94 \mathrm{GHz}$ and $7.38 \mathrm{GHz}$, respectively. Gain exceeds $8.85 \mathrm{~dB}$ and $6.59 \mathrm{~dB}$ for $4.94 \mathrm{GHz}$ and $7.38 \mathrm{GHz}$, respectively.

\section{Acknowledgement}

We would like to thank the Sonnet software team for providing us with a license which gave us the ability to work from home during the pandemic of coronavirus.

\section{References}

[1] S. S. Ferouani, Z. Z. Bendahmane and A. A. T. Ahmed, "Design and analysis of dual star shape slotted patch antenna", June 2017.

[2] G. Pravin, A. K. Rastogi, "Design and analysis of the single and dual fed patch antenna array", Department of Physics and Electronics, Barkatullah University Bhopal (M.P.), India, 2018 Jetir, vol. 5 , no. 2, 2018 .

[3] Sonnet Suites, ver. 17.52. Szracuse, New York

[4] S. T. Imeci, "Design and manufacture of patch antennas", International University of Sarajevo, Sarajevo 2017

[5] A. Khidre, K.-F. Lee, F. Yagn, and A. Z. Elsherbeni, "Circular polarization reconfigurable wideband E-shaped patch antenna for wireless applications," Antennas and Propagation, 2013

[6] A. Kumar, P. Kumar, A. Deb, An $\xi$-shaped microstrip antenna with unidirectional propagation, Accepted for the publication in the proceedings of ICCSP:15, Melmaruvathur, 2015

[7] Hermann J., Zach D. and Angel A. Analysis and design of ISM-band patch antenna array, Georgia Institute of Technology, USA, 2012

[8] P.Li, J. Liang, and X. Chen, Study of printed elliptical/circular slot antennas for ultrawideband applications, 2012

[9] A. Bhattacharya, R. Jyoti, Frequency Reconfigurable Patch Antenna Using PIN Diode at X-Band IEEE 2nd International Conference on Recent Trends in Information Systems , 2015

[10] Shanmuganatham, T. and Deepanshu Kaushal, Design of multi utility multi band microstrip calculator shaped patch antenna using coaxial feed, Communication and Signal Processing (ICCCSP), 2017 International Conference on. IEEE, 2017

[11] M. Mishra and J. M. Singh, 'Design of triangular microstrip patch antenna array for 4G (LTE) applications', International Journal of Software \& Hardware Research in Engineering, 2017.

[12] A. Choudhary, P. Kumar, A. Kumar, "Design and analysis of microstrip Patch antenna with elliptical slot," Discovery, vol.42, no.193, pp. 113-116, 2015. 